Mitteilungen der Österreichischen Geographischen Gesellschaft, 160. Jg., S. 398-402

(Annals of the Austrian Geographical Society, Vol. 160, pp. 398-402)

Wien (Vienna) 2018, https://doi.org/10.1553/moegg160s398

\title{
Albert Hofmayer - eine Würdigung zUM 70. Geburtstag
}

\author{
Wolfgang SchWARZ und Christian StAUdACHER, beide Wien*
}

mit 1 Abb. im Text

\begin{abstract}
Albert HOFMAYER, akademischer Lehrer und Forscher auf dem Gebiet der Wirtschaftsgeographie und engagiertes Vorstandsmitglied der Österreichischen Geographischen Gesellschaft (ÖGG), feiert in diesem Jahr seinen 70. Geburtstag. Er wurde als Sohn des Gerbermeisters und Glasers Adalbert und der Einzelhandelskauffrau Margarete HofMAYER am 15. September 1948 in Aschbach-Markt im niederösterreichischen Mostviertel geboren und besuchte das Stiftsgymnasium Seitenstetten, wo er 1966 mit Auszeichnung maturierte.

Sein dortiger Klassenvorstand, Prof. Franz Urban, vermochte ihn für Geographie und Geschichte zu begeistern. Bereits im Volksschulalter hatte HOFMAYER Landkarten, Stadtpläne und Atlanten eifrig studiert und sich ein beachtliches topographisches Wissen angeeignet. Er studierte daher an der Universität Wien Geographie und Geschichte (Lehramt). Zu seinen wichtigsten akademischen Lehrern zählt er die Geographen Hans BobeK, Hans Spreitzer, Erik Arnberger und Julius Fink sowie die Historiker Heinrich Lutz und Günther Hamann. Seine Dissertation im
\end{abstract}

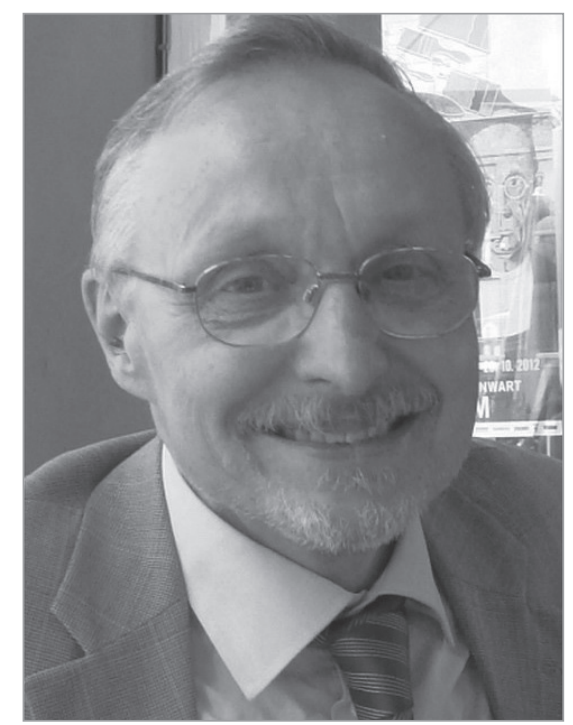

Albert HOFMAYER

\footnotetext{
* Wirkl. Hofrat i.R. Univ.-Doz. Dr. Wolfgang Schwarz, Mitglied des Vorstands der ÖGG, Pappelweg 1/26, A-1220 Wien; Ao. Univ.-Prof. i.R. Dr. Christian Staudacher, Vizepräsident der ÖGG, Seitenberggasse 20/30,A-1160 Wien; E-Mail: wolfgangschwarz@inode.at; christian.staudacher@sdvm.de
} 
Umfang von 303 Seiten behandelt „Die viehlose Landwirtschaft im Marchfeld“, ein Thema, das ihm Hans BoBeK vorgeschlagen hatte. 1974 wurde Albert HOFMAYER zum Dr. phil. promoviert. Im Folgenden soll sein Wirken als Geograph, gegliedert in drei Etappen seiner Karriere, dargestellt und bewertet werden.

\section{Akademie der Wissenschaften - Mitarbeiter von Hans Bobek}

Gleich nach seiner Promotion nahm HofMAYER auf Einladung von Hans BobeK seine Tätigkeit als wissenschaftlicher Angestellter der Österreichischen Akademie der Wissenschaften (ÖAW) in der „Kommission für Raumforschung“ auf, wo er Josef Steinbach, dem späteren Professor für Wirtschaftsgeographie an der Katholischen Universität Eichstätt, nachfolgte. Maria BoBEK-FesL, Gertrude Mras und seit 1981 auch Heinz FassmanN, später Universitätsprofessor für Angewandte Geographie, Raumforschung und Raumordnung an der Universität Wien, waren die Kolleginnen und Kollegen während seiner Zeit an der „Akademie“.

In den Jahren von 1974 bis 1983 war Albert HOFMAYER an der ÖAW mit dem Entwurf und der Bearbeitung thematischer Karten für den „Atlas der Republik Österreich“ und damit zusammenhängenden Textpublikationen beschäftigt. 1981 veröffentlichte er gemeinsam mit Hans BoBEK das grundlegende Werk „Gliederung Österreichs in wirtschaftliche Strukturgebiete“, eine eingehende Interpretation der entsprechenden Karte im Österreich-Atlas. Mithilfe von Daten über die sektorale Wirtschaftsstruktur, die Pendlerbilanz und die Tourismusintensität wurde das Bundesgebiet in rund 200 wirtschaftsräumliche Einheiten untergliedert, die jeweils einem von neun signifikanten wirtschaftlichen Strukturtypen zugeordnet wurden. Drei Jahrzehnte später wurde diese Untersuchung anhand aktueller Daten von Huberta KulmHOFER, einer Dissertantin HofMaYERS, wiederholt. Ein Vergleich der beiden Karten über das wirtschaftsräumliche Mosaik innerhalb Österreichs lässt erkennen, welche gravierenden Veränderungen sich im Zeitraum von 1971 bis 2001 abgespielt haben, etwa das völlige Verschwinden des Raumtyps „Agrargebiete“ zugunsten diverser ländlicher „Menggebiete“ sozioökonomischer Art mit oftmals hohen Auspendlerüberschüssen (vgl. dazu den Beitrag von Kulmhofer und Hofmayer in den MÖGG, Band 153, 2011). Die beiden Wirtschaftsraum-Karten hängen übrigens in den Räumlichkeiten des renommierten Wirtschaftsforschungsinstitutes „Economica“(Wien) - ein Beweis für ihre besondere Qualität.

\section{Wirtschaftsuniversität Wien - Forscher, Lehrer und Dienstleister}

Die wissenschaftliche Laufbahn Albert HofMAYERs hat mit seinem Eintritt in das Institut für Wirtschaftsgeographie der Wirtschaftsuniversität Wien (Prof. Erhard WinKLER, Prof. Karl SinNHUBER) im Jahr 1983 eine Neuorientierung zur Wirtschaftsgeographie erfahren, die sich dann ab 1991 in der „Abteilung für Angewandte Regional- und Wirtschaftsgeographie“ (ARWI) noch vertieft hat. Dies zeigt sich an seinen Schwerpunkten in der Lehrtätigkeit, die durch Proseminare und Seminare zur Wirtschaftsgeographie, Übungen zur Forschungsmethodik, zu statistischen Methoden sowie zur EDV-Kartographie und GIS gekennzeichnet war. Ab seiner Habilitation 2006 und der Ernennung zum Außerordentlichen Universitätsprofessor hielt HoFMAYER auch Vorlesungen zur Wirtschaftsgeographie des Weltwirtschaftsraumes. Dazu kam die Betreuung von ca. 300 Diplomarbeiten und fünf Dissertationen. Seine Lehrtätigkeit und der Umgang mit den Studierenden waren immer von Hilfsbereitschaft und großer Ausdauer gekennzeichnet. Wann auch immer man an seiner Zimmertür vorbeikam: Dort warteten fast immer Studierende, früh am Morgen oder auch spät am Abend. Wegen des Andranges mussten sie oft länger ausharren, aber sie wurden immer intensiv betreut 
und beraten: Das reichte von längeren Fachgesprächen bis zur geduldigsten Beratung über die Datenauswertung, die Literatursuche usw. Hofmayer äußerte sich dazu: „Ich fasse meine Arbeit als Dienstleistung für die Studierenden auf".

Am Institut für Wirtschaftsgeographie der WU Wien und an dessen Nachfolge-Institut für Wirtschaftsgeographie und Geoinformatik (Prof. Manfred M. FISCHER), wo er auch ab 2009 EDV-Verantwortlicher und stellvertretender Institutsvorstand war, und besonders in der weitgehend selbstständig agierenden „Abteilung für Angewandte Regional- und Wirtschaftsgeographie“ (ARWI; Leitung Christian STAUDACHER) war er in der Verwaltung, bei Forschungsprojekten usw. ein vielbeschäftigter Mitarbeiter: In der alltäglichen Zusammenarbeit war er in vielen Sachbereichen der inneren und äußeren Organisation eingespannt, insbesondere auch als Verantwortlicher für die EDV-Ausstattung der Abteilung. Hier hat er nicht nur die Studierenden (siehe oben), sondern auch die Mitarbeiter/ innen der Abteilung intensiv betreut. Diese Expertise und seine Fachkenntnis und Erfahrung im Umgang mit statistischen Methoden und EDV-Kartographie machten ihn zu einem unverzichtbaren Mitarbeiter bei Forschungsprojekten, zum Beispiel bei dem Abteilungsprojekt „Kaufkraftstromanalysen Niederösterreich 1988 und Wien 1991“ (Projektleiter Klaus ARNOLD), beim Projekt „Bahnhof und Stadt“ 2000-2002 oder beim Forschungsschwerpunkt „Dienstleistungsgeographie“, insbesondere bei den Forschungen zur Bedeutung der Wirtschaftsdienste (vgl. dazu die Habilitation von Christian STAUDACHER).

Im beruflichen Zusammenleben war HOFMAYER immer ein ungewöhnlich hilfsbereiter Mensch und stiller Arbeiter, der sich nie in den Vordergrund drängte. Er hat damit wesentlich zum kollegialen Klima und zum offenen Führungsstil an der ARWI beigetragen. Manchmal hatte ich (STAUDACHER) als Abteilungsleiter das Gefühl, ich müsste Albert zu ein bisschen mehr Egoismus drängen, vor allem dann, wenn es um seine eigene wissenschaftliche Karriere ging.

Obwohl in HofmaYers Forschungen und Lehrveranstaltungen an der Wirtschaftsuniversität Wien Theorie und Empirie einen hohen Stellenwert einnahmen, war es ihm stets ein großes Anliegen, die konkrete lebens- und wirtschaftsräumliche Realität, also die Staaten, Regionen und Siedlungen in ihrer Vielfalt, näher kennenzulernen und dies seinen zahlreichen Schülerinnen und Schülern zu vermitteln. Dazu dienten die zahlreichen Exkursionen. HofmaYer nahm im Zeitraum von 1984 bis 2013 an 16 großen Auslandsexkursionen teil, acht davon fanden unter seiner fachlichen und organisatorischen Leitung statt. Besonders beeindruckt war er, damals noch als junger Assistent, von den Exkursionen in die ehemalige Sowjetunion (1984) sowie nach Mexiko und Kuba (1985), beide geleitet von Karl SinNHUBER. Geradezu „legendär“ waren die vier Exkursionen, die in die Zentren der Europäischen Union und in deren umliegende Regionen führten. So wurden 2009 die EU-Metropolen Straßburg (Europäisches Parlament), Frankfurt am Main (Europäische Zentralbank) und Luxemburg (Eurostat) besucht, aber auch das Technologiezentrum Karlsruhe, die Stadtplanung von Frankfurt am Main, ein Weingut an der Mosel, eine historische Eisenhütte in Völklingen sowie die Altstädte von Trier, Metz, Nancy und Straßburg (Leitung Hofmayer, Schwarz, StAudacher) ein unvergessliches Erlebnis. Die Organisation dieser und anderer Exkursionen funktionierte dank HOFMAYERs präziser Planung perfekt.

\section{Wirtschaftsgeograph mit breiter humangeographischer Perspektive}

Die Forschungstätigkeit von Albert HOFMAYER zeichnet sich, im Kontext einer von ihm inhaltlich breit verstandenen Wirtschaftsgeographie, durch eine besondere Themenvielfalt aus. Anknüpfend an seine Dissertation befasste er sich weiterhin mit agrargeographischen Fragen (Artikel „Das agrarische Nutzungspotenzial - eine vernachlässigte Größe“, 1988). Es folgten tourismuswirtschaftliche Studien, gipfelnd in der clusteranalytisch-kartographischen Arbeit über die „Typisierung von 
Fremdenverkehrsgemeinden in Österreich“ (gem. mit Felix JüLG, 1989). Wichtig sind auch seine Studien zum Themenfeld der Ressourcengeographie unter einem humanökologischen Aspekt und die von einem humanistisch-religiösen Zugang her betriebenen Arbeiten zur Weltbevölkerungsentwicklung (Artikel „Ein halbes Jahrhundert Weltbevölkerungsentwicklung“, 2001), bei denen es um „Fragen der bevölkerungsgeographischen Regionalisierung der Erde“ ging, insbesondere auch um die Tatsache der Zunahme der Weltregionen und Staaten mit einer „Fruchtbarkeit unter dem Generationenersatzniveau (,replacement level')“.

Sein umfangreichster Forschungsbereich ist die Thematik des Einkaufsverhaltens, insbesondere im ländlichen Raum: Dies begann 1990 mit einer Arbeit über den Einfluss von Strukturvariablen auf das räumliche Muster des Einkaufsverhaltens und reichte über seine Habilitationsschrift (siehe unten) bis zu regionalen Studien zu diesen Fragestellungen, z. B. im Bezirk Amstetten (2001) und in Bahnhofsvierteln in österreichischen und europäischen Städten (2002).

Die zentrale Arbeit dazu ist die Habilitationsschrift von Albert HofMAYER (1995) mit dem Titel „Grundbedarfsdeckung im ländlichen Raum in Abhängigkeit von Wohnstandorts- und Haushaltsmerkmalen“, eingereicht an der Universität Salzburg, und in gedruckter Form in den Wiener Geographischen Schriften, Band 62, 1997, erschienen. Dabei handelt es sich um eine multivariate, statistisch schließende Untersuchung auf breiter empirischer Basis mit einem wohlüberlegten und argumentierten Variablenspektrum. Schon von der Themenformulierung her befasst sie sich mit den Grundbedürfnissen der alltäglichen Versorgung und bewertet, wie einerseits Strukturmerkmale (Familiengröße, Kinderzahl, Einkommen, Mobilität, ...) und andererseits „,räumliche“ Situationen (Wohnstandort und damit gegebene und erreichbare Angebote) diese alltägliche Versorgung beeinflussen, sie leicht machen oder eben auch wesentlich erschweren können. Trotz einer auf dem ersten Blick recht statistisch anmutenden Analyse zeigt sich eine immer wieder in den wissenschaftlichen Fragestellungen von HOFMAYER auftauchende engagierte, gleichsam „sozial“-geographische Haltung im Sinn einer „Engagierten Geographie“ (Martin BoEsCH). Mit dieser Arbeit hat HofmaYer deutlich belegt, dass er nach einem zehnjährigen Verfahren 2006 längst „,habilitationsreif“ war, was er in seinem weiteren Berufsleben als Lehrer und Forscher wirkungsvoll bewiesen hat.

Großen Beifall, etwa vom Wirtschaftsgeographen Wigand RitTER, hat schließlich HofMAYERS Artikel „,Geographische“ Einflussfaktoren in aktuellen Modellen der Wirtschaftsentwicklung“ (Wirtschaftsgeographische Studien, Band 34/35, Wien 2008) gefunden. In der für ihn typischen akribischen Art unternahm er eine Literaturauswertung auf der Basis von sechs großen Datenbanken über sämtliche 1992 bis 2007 erschienenen Arbeiten (insgesamt 1.300) zu dieser Thematik. In seiner kritischen Bewertung dieser Publikationen bemängelt er die zumeist willkürliche und kaum theoriebezogene Auswahl solcher „geographischer“ Einflussgrößen, besonders bei den Modellrechnungen der „New Economic Geography“. Als Reaktion darauf entwarf HOFMAYER einen eigenen konzeptionellen Rahmen zur Analyse der geographischen Voraussetzungen der Wirtschaftsentwicklung von Staaten, der - im Gegensatz zu den meisten wirtschaftswissenschaftlichen Studien - auch die demographischen Aspekte und Humanressourcen mitberücksichtigt.

Die obigen Kurzbeschreibungen wichtiger wirtschaftgeographischer Arbeiten von Albert HoFMAYER lassen erkennen, welche Stärken ihn als Wissenschaftler besonders auszeichnen: Seine Neugierde an gesellschaftlich, ökonomisch und ökologisch wesentlichen Themen und seine Fähigkeit, dabei forschungsrelevante Fragestellungen zu erkennen und abzuhandeln; gründliche topographische Kenntnisse; klarer Theoriebezug und präzise Terminologie in seinen Forschungsarbeiten, hohe Qualität der Empirie: Akribische Literaturrecherchen, Anwendung anspruchsvoller Methoden der Statistik und kartographischen Darstellung; besondere Begabung für Kategoriebildungen und Klassifizierungen, mit denen er neue, innovative Wege beschritten hat. Daneben kommen ihm bei seinen wissenschaftlichen Arbeiten und Vorträgen (z. B. beim IGU-Weltkongress 2004 in Glasgow) seine ausgezeichneten Fremdsprachenkenntnisse (Englisch, Spanisch) sehr zugute. 


\section{„Stütze“6 wissenschaftlicher Vereinigungen (ÖGW, ÖGG)}

Sehr beachtlich und deshalb besonders erwähnenswert ist auch das altruistische, da ehrenamtliche Engagement von Albert HOFMAYER in wissenschaftlichen Vereinigungen:

Die „Österreichischen Gesellschaft für Wirtschaftsraumforschung“ (ÖGW), gegründet 1962 an der damaligen Hochschule für Welthandel (Institut für Geographie, Prof. Leopold ScHEIDL), war am Institut für Wirtschaftsgeographie und dann an der ARWI ein völlig integriertes Instrument der Förderung der Wirtschaftsgeographie in Forschung und Lehre. Die gemeinsam von der ÖGW und der ARWI über viele Jahre durchgeführte Vortragsserie „Kolloquium Raum und Wirtschaft“ hat Albert HOFMAYER auch nach der Auflösung der ÖGW 2009 bis heute als Lehrbeauftragter der Wirtschaftsuniversität Wien weitergeführt. In der ÖGW hat er sich während seiner gesamten Dienstzeit intensiv engagiert. Zunächst als persönlicher Assistent der professoralen Präsidenten (SINNHUBER, WINKLER) und danach als Generalsekretär betreute er die Mitgliederdatei, die zahlreichen Aussendungen und Veranstaltungen und seit 1985 fast alle Hefte der Reihe „Wirtschaftsgeographische Studien“ als Schriftleiter. Gerade diese Leistung war, wie in allen anderen dargestellten Bereichen, von Sorgfalt, Genauigkeit und hohem zeitlichem Einsatz geprägt.

In der „Österreichischen Geographischen Gesellschaft“ (ÖGG), der Albert HOFMAYER seit 1974 als Mitglied angehört, ist er seit 2011 Mitglied des Vorstandes, weil er die Funktion des Geschäftsführers des „Geographie-Fonds der ÖGG“ übernommen hat. Dieser Fonds wurde 2012 auf der Basis von Spenden und der eigenständigen Kapitalbildung und -widmung der ÖGG eingerichtet und fördert über die jährliche Ausschreibung von inzwischen vier Preisen wissenschaftliche Leistungen im Bereich der Geographie. Die Verwaltung eines solchen Fonds erfordert größte Umsicht, bei der auch die eingerichtete Jury gefordert ist, und macht viel Arbeit: Für die derzeit ausgeschriebenen Preise sind beispielsweise bis zum Jahresende 2018 insgesamt 32 Arbeiten eingereicht worden, was bedeutet, dass vom Geschäftsführer eine große Zahl von Gutachterinnen und Gutachtern gefunden und betreut werden muss, damit dann die Jury im nächsten Oktober über die Vergabe der Preise entscheiden kann. Dies ist eine wahre Sisyphusarbeit, die außergewöhnlichen „Altruismus“"verlangt und die in der nötigen Qualität fast nur von Albert HOFMAYER geleistet werden kann. Die ÖGG kann da einfach nur „Danke“ sagen!

Viele werden sich fragen, was unserem Jubilar die Kraft für seine anspruchsvollen Leistungen in Forschung, Lehre und Vereinstätigkeit gibt. Einen starken Rückhalt verleiht ihm zweifellos sein intensiver römisch-katholischer Glaube. Darauf angesprochen, antwortet er mit den knappen, aber überzeugenden Worten „Der Glaube ist eine Gnade“. Besonders bestärkt darin hat ihn der charismatische spanische Priester Josefmaria EsCRIvá, der später heiliggesprochen wurde und den HOFMAYER schon in seiner Studienzeit persönlich kennengelernt hat.

Albert HOFMAYER war und ist ein maßgeblicher Wissenschaftler, ein großartiger akademischer Lehrer und eine bedeutsame Stütze der Österreichischen Geographischen Gesellschaft. Darüber hinaus ist er uns allen ein großes Vorbild an Menschlichkeit. Seine besonnene, unaufgeregte Art, die Bereitschaft, seinem Gegenüber geduldig zuzuhören und seine Sorgen ernst zu nehmen, seine Empathie und Hilfsbereitschaft beeindrucken uns immer wieder von Neuem.

Wir wünschen Dir, lieber Albert, und auch uns, dass dies noch lange so bleiben möge. Alles Gute zu Deinem „Siebziger“ und noch viele schöne Jahre! 